\title{
Épocas de aplicação e concentrações de ácido abscísico no incremento da cor da uva 'Isabel'
}

\section{Application time and concentrations of abscisic acid on the color development of 'Isabel' grapes}

\author{
Renata Koyama ${ }^{1}$; Lilian Yukari Yamamoto ${ }^{1}$; \\ Wellington Fernando Silva Borges ${ }^{2}$; Marina Bonini Pascholati ${ }^{3}$; \\ Rogério de Sá Borges ${ }^{4}$; Adriane Marinho de Assis ${ }^{5}$; Sérgio Ruffo Roberto ${ }^{6 *}$
}

\section{Resumo}

A uva 'Isabel', principal cultivar utilizada para a elaboração de suco no Brasil, apresenta deficiência de cor, sendo uma das alternativas, a aplicação do ácido abscísico ( $S$-ABA), visto que o acúmulo de antocianinas, pigmento que interfere na cor das bagas, parece ser regulado, por este fitohormônio. $\mathrm{O}$ objetivo do trabalho foi avaliar o efeito de concentrações de $S$-ABA, aplicadas em épocas distintas nos cachos da videira 'Isabel', na melhoria dos atributos de cor. As videiras foram conduzidas em espaldeira, no espaçamento de $2 \times 1 \mathrm{~m}$, em cordão esporonado. $\mathrm{O}$ delineamento experimental utilizado foi em blocos casualizados, com quatro repetições e cinco tratamentos: Testemunha; $S$-ABA $200 \mathrm{mg}$ $\mathrm{L}^{-1}$ aplicado sete dias após o veraison (DAV); $S$-ABA $400 \mathrm{mg} \mathrm{L}^{-1}, 7 \mathrm{DAV}$; $S$-ABA $200 \mathrm{mg} \mathrm{L}^{-1}, 7 \mathrm{DAV}+$ $200 \mathrm{mg} \mathrm{L}^{-1}, 10$ dias antes da colheita (DAC); e $S$-ABA $400 \mathrm{mg} \mathrm{L}^{-1}, 7 \mathrm{DAV}+400 \mathrm{mg} \mathrm{L}^{-1}, 10 \mathrm{DAC}$. No momento da colheita foram avaliadas as variáveis massa e diâmetro das bagas, massa e comprimento dos cachos, teor de sólidos solúveis (SS), acidez titulável (AT) e índice de maturação (SS/AT). Foi avaliado, também, a concentração de antocianinas e o índice de polifenóis totais do mosto e do suco, elaborado pelo método da panela extratora e a cor das bagas por colorimetria. A aplicação de $S$-ABA não influenciou as características físicas das uvas, entretanto, propiciou o aumento no teor de SS e a relação SS/AT das bagas, exceto para a concentração de $200 \mathrm{mg} \mathrm{L}^{-1}$ aplicada sete dias após o veraison. O $S$-ABA exerce efeito sobre a melhoria no teor de antocianinas das bagas e do suco da uva 'Isabel', principalmente na dose de $400 \mathrm{mg} \mathrm{L}^{-1}$ aos $7 \mathrm{DAV}+10 \mathrm{DAC}$, além de propiciar a melhoria no atributo cor das bagas.

Palavras-chave: Vitis labrusca, suco de uva, antocianina

\begin{abstract}
The grape 'Isabel' main cultivar used for juice production in Brazil has a deficiency of coloring and an alternative is the application of abscisic acid (S-ABA), since the accumulation of anthocyanins,
\end{abstract}

\footnotetext{
${ }^{1}$ Eng $^{\text {as }}$ Agras $^{\text {as }}$ Discentes do Curso de Doutorado em Agronomia, Fitotecnia, Universidade Estadual de Londrina, UEL, Londrina, PR.E-mail: emykoyama@hotmail.com,domotokoichitsuyoshi@hotmail.com

${ }^{2}$ Eng $^{\circ}$ Agr $^{\circ}$, Discente do Curso de Mestrado em Agronomia, Fitotecnia, UEL, Londrina, PR. E-mail: wellingtonfernando@msn. com

${ }_{3}$ Discente do curso de Agronomia, UEL, Londrina, PR. E-mail: ma_pascholati@hotmail.com

${ }^{4} \mathrm{Eng}^{\mathrm{o}} \mathrm{Agr}^{\mathrm{o}}$, Dr. em Fitotecnia, UEL, Analista de Produtos e Mercado da Empresa Brasileira de Pesquisa Agropecuária, Londrina, PR. E-mail: rdsborges@terra.com.br

${ }^{5}$ Eng $^{\text {a }}$ Agr $^{\mathrm{a}}$, Prof ${ }^{\mathrm{a}}$. Dr. Adjunta, Dept ${ }^{\mathrm{o}}$ de Agronomia, Universidade Federal de Pelotas, UFPEL, Pelotas, RS. E-mail: agroadri@ ig.com.br

${ }^{6}$ Eng $^{\circ}$ Agr $^{\circ}$, Prof. Dr. Associado, Dept ${ }^{\circ}$ de Agronomia, Centro de Ciências Agrárias, UEL, Londrina, PR. E-mail: sroberto@uel.br

* Autor para correspondência
} 
pigment that interferes in the color of berries, appears to be regulated by this growth regulator. The aim of this research was to evaluate the effect of different concentrations of $S$-ABA applied at different times in the clusters of 'Isabel' grapes to improve their color attributes. The vines were conducted in a vertical support structure, spaced $2 \times 1 \mathrm{~m}$ in cordon. A randomized block design was used as a statistical model, with 4 replications and 5 treatments, as follows: control; $S$-ABA $200 \mathrm{mg} \mathrm{L}^{-1}$ applied seven days after veraison (DAV); S-ABA $400 \mathrm{mg} \mathrm{L}^{-1} 7 \mathrm{DAV}$; $S$-ABA $200 \mathrm{mg} \mathrm{L}^{-1} 7 \mathrm{DAV}+S$-ABA 200 $\mathrm{mg} \mathrm{L}^{-1} 10$ days before harvest (DBH); S-ABA $400 \mathrm{mg} \mathrm{L}^{-1} 7 \mathrm{DAV}+400 \mathrm{mg} \mathrm{L}^{-1} 10 \mathrm{DBH}$. At harvest the following variables were evaluated: mass and diameter of the berries, mass and length of the clusters; soluble solids (SS), titratable acidity (TA) and maturation index (SS/TA). It was also evaluated the concentration of anthocyanins and total polyphenol index in wine and juice, prepared by the extraction method of the pan and color of the berries by colorimetry. The application of $S$-ABA did not influence the physical characteristics of the grapes, however, favored the increase of SS and SS/TA of the berries, except for the concentration of $200 \mathrm{mg} \mathrm{L}^{-1}$ applied seven days after veraison. The $S$-ABA has an effect on improving the content of anthocyanins of the berries and the juice of the 'Isabel' grape, mainly in the $400 \mathrm{mg} \mathrm{L}^{-1}$ applied $7 \mathrm{DAV}+10 \mathrm{DBH}$, besides enabling the improvement in the color attribute of berries.

Key words: Vitis labrusca, grape juice, anthocyanins

\section{Introdução}

O Paraná é um dos principais estados produtores de uva, destacando-se duas regiões vitivinícolas importantes: a metropolitana de Curitiba e o norte do estado, onde tradicionalmente predomina a produção de uvas finas para consumo in natura. Entretanto, nos últimos anos, tem-se verificado a diversificação da produção, com a introdução de cultivares de uvas americanas e híbridas voltadas para a elaboração de suco e vinho de mesa (MELLO, 2012; KISHINO; CARVALHO; ROBERTO, 2007).

Segundo Protas e Camargo (2011), a produção de uvas para a elaboração de sucos é uma alternativa promissora, por possibilitar a diversificação da produção sem muitas alterações nos sistemas de cultivo. Em decorrência de projetos para a produção de suco de uva concentrado, implantados por cooperativas agroindustriais, observa-se o crescimento na produção de uvas para este fim, com o plantio das cultivares Isabel (Vitis labrusca), Concord, Bordô, BRS-Carmem e BRS-Rúbea.

Quanto às características das uvas destinadas a elaboração de suco, o maior interesse das indústrias de suco do norte do Paraná é por cultivares de maturação tardia, uma vez que a produção do suco de uva é complementar à do suco de citros. Desta forma, a uva deve estar disponível para a indústria após o término da safra de laranja, que se estende até o mês de dezembro (SATO et al., 2008; PROTA; CAMARGO, 2011). Dentre as cultivares, a uva 'Isabel' é a base para o suco brasileiro, pois apresenta boa adaptação às condições climáticas do Sul do Brasil e é altamente fértil, com produções abundantes. É a mais plantada em estados como o Rio Grande do Sul e Santa Catarina; embora o suco elaborado com esta cultivar apresente deficiência em cor, devido à falta de pigmentos (MAIA; CAMARGO, 2007).

A cor da uva está relacionada à antocianina, pigmento que confere cor aos frutos, flores e legumes, participando da impregnação de lignina nas paredes pecto-celulósicas (RIBICHAUD; NOBLE, 1990). As antocianinas são parte dos compostos flavonoides das uvas tintas, sendo uma das características mais relevantes na produção de suco e vinho de alta qualidade. Sabe-se que a expressão das antocianinas depende de fatores internos, como o regulador vegetal ácido abscísico ( $S$-ABA), que induz o fator de transmissão MYB1A, proteína encarregada de regular a transmissão de genes que compõem a rota biosintética das antocianinas das uvas tintas (JEONG et al., 2004). Vários trabalhos sugerem que aplicações exógenas de $S$-ABA proporcionam o aumento no teor de antocianinas na casca de uvas (PEPPI; FIDELIBUS; DOKGOZLIAN, 2006; PEPPI; FIDELIBUS; DOKOOZLIAN, 2007a; 
CANTÍN; FIDELIBUS; CRISOTO, 2007; PEPPI; FIDELIBUS, 2008; ROBERTO et al., 2012, 2013; KOYAMA et al., 2014).

Quanto mais intensa a cor da uva, mais interessante se torna do ponto de vista funcional e do processamento, devido ao fato de que as uvas de cor escura apresentam maior conteúdo de compostos fenólicos, além da capacidade antioxidante, anticarcinogênica e antiviral, não havendo a necessidade de cortes com outros sucos elaborados a partir de uvas tintureiras (ABE et al., 2007; JUBILEU; SATO; ROBERTO, 2010).

Em vista desses aspectos, o objetivo deste estudo foi avaliar o efeito de concentrações de $S$-ABA, aplicadas em épocas distintas, na melhoria dos atributos da cor de cachos da videira 'Isabel'.

\section{Material e Métodos}

O experimento foi conduzido durante a safra regular da videira Isabel (Vitis labrusca), em um vinhedo de 6 anos, formado de pé franco, localizado na Fazenda Escola da Universidade Estadual de Londrina - PR, $\left(23^{\circ} 22^{\prime} \mathrm{S}, 51^{\circ} 10^{\prime} \mathrm{W}\right.$; altitude 585 $\mathrm{m})$. O solo é um Latossolo Vermelho Distroférrico, com $630 \mathrm{~g} \mathrm{~kg}^{-1}$ de argila, $180 \mathrm{~g} \mathrm{~kg}^{-1}$ de silte e 190 $\mathrm{g} \mathrm{kg}^{-1}$ de areia na camada de $0-25 \mathrm{~cm}$. O clima é classificado, segundo Köppen, como $C f a$, com precipitação principalmente na primavera e verão, com média anual de $1.610 \mathrm{~mm}$. A temperatura média anual é de $21,2^{\circ} \mathrm{C}$ e a umidade relativa média de 70,7\% (CAVIGLIONE et al., 2000).

As plantas foram conduzidas sob o sistema espaldeira em cordão esporonado e, no espaçamento de $2 \mathrm{~m}$ entre fileiras e $1 \mathrm{~m}$ entre plantas. A poda de frutificação foi realizada em julho de 2011, deixando-se uma gema por esporão, e aplicado em seguida cianamida hidrogenada a $2,5 \%$ na gema apical, para a indução da brotação e uniformização. Foi avaliado o efeito do ácido abscísico $S$-ABA (100 $\mathrm{g} \mathrm{L}^{-1}$ de ingrediente ativo) aplicado em diferentes concentrações e, no caso dos tratamentos d e e, em duas épocas, conforme segue: a) Testemunha; b) $S$-ABA $200 \mathrm{mg} \mathrm{L}^{-1}$ aplicado sete dias após o veraison (DAV); c) $S$-ABA $400 \mathrm{mg} \mathrm{L}^{-1}, 7 \mathrm{DAV}$; d) $S$-ABA $200 \mathrm{mg} \mathrm{L}^{-1}, 7 \mathrm{DAV}+200 \mathrm{mg} \mathrm{L}^{-1}, 10$ dias antes da colheita (DAC); e e) $S$-ABA $400 \mathrm{mg} \mathrm{L}^{-1}$, $7 \mathrm{DAV}+400 \mathrm{mg} \mathrm{L}^{-1}, 10 \mathrm{DAC}$. Foi adicionado à calda de todos os tratamentos o espalhante nãoiônico BreakThru ${ }^{\circledR}\left(0,3 \mathrm{~mL} \mathrm{~L}^{-1}\right)$. O delineamento experimental foi em blocos casualizados, com cinco tratamentos e quatro repetições, sendo cada parcela composta por 10 plantas. Foram marcados, previamente à aplicação dos produtos, 10 cachos uniformes em cada parcela.

Para a aplicação dos tratamentos, os cachos foram pulverizados no período da manhã, utilizando-se pulverizador costal com pressão de 40

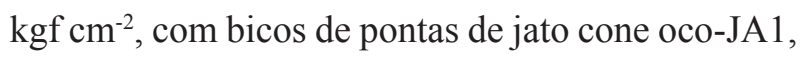
proporcionando cobertura completa e uniforme, sendo empregado volume de calda de $800 \mathrm{~L} \mathrm{ha}^{-1}$.

Foram realizados os tratos culturais habitualmente empregados na região em relação à nutrição, controle de plantas daninhas, manejo de pragas e de doenças. Na colheita, de cada cacho marcado foram coletadas três bagas para avaliações físico-quimicas, sendo retirada uma baga da porção superior, mediana e inferior, totalizando 30 bagas por parcela. Os cachos de cada parcela foram colhidos manualmente, com auxílio de uma tesoura de poda, e submetidos à limpeza. Após a colheita foi extraído o suco das uvas pelo método da panela extratora, para a realização das análises químicas.

Ascaracterísticasfísico-químicas foramavaliadas mediante determinação da massa (g) e diâmetro das bagas $(\mathrm{mm})$, medido com um paquímetro digital, massa $(\mathrm{kg})$ e comprimento dos cachos $(\mathrm{cm})$. Quimicamente as bagas foram avaliadas quanto ao teor de sólidos solúveis - SS ( ${ }^{\circ}$ Brix), acidez titulável (AT) (\% de ácido tartárico) e relação SS/AT. As análises químicas foram realizadas no Laboratório de Análise de Frutas, pertencente ao Centro de Ciências Agrárias da Universidade Estadual de Londrina-PR. O teor de SS foi determinado em 
refratômetro digital de bancada com compensação automática de temperatura (Modelo DR301-95, Krüss Optronic, Alemanha), por meio da trituração das bagas, e o resultado expresso em ${ }^{\circ}$ Brix. A determinação da AT foi realizada por titulação do mosto com solução padronizada de $\mathrm{NaOH} 0,1 \mathrm{~N}$, em titulador semiautomático, adotando como ponto final da titulação o $\mathrm{pH}=8,2$, e o resultado expresso em porcentagem de ácido tartárico (INSTITUTO ADOLFO LUTZ, 1985).

Além das características físico-químicas, foram analisados a concentração de antocianinas e polifenóis totais (PT) do mosto e do suco. Para tanto, no momento da colheita, foram coletadas 60 bagas de cada parcela, sendo estas retiradas da parte superior, mediana e basal dos cachos.

Para a avaliação do teor de antocianinas, a extração foi realizada segundo a metodologia de Lee e Francis (1972), com algumas modificações: foram pesadas $50 \mathrm{~g}$ de cada amostra de uva e trituradas durante dois minutos com $50 \mathrm{~mL}$ da solução solvente de etanol 70\%. Posteriormente, foi completado com o solvente até o volume de 200 $\mathrm{mL}$ e armazenada a solução coberta com parafilme durante 12 horas, a $4^{\circ} \mathrm{C}$. O material foi filtrado em funil de Büchner com auxílio de bomba de vácuo, foram retirados $100 \mathrm{~mL}$ do filtrado e completado com álcool $70 \%$ até o volume $200 \mathrm{~mL}$. Desta, foram retiradas amostras de $2 \mathrm{~mL}$, completadas até o volume de $100 \mathrm{~mL}$, e deixadas por duas horas no escuro. A leitura foi realizada em espectrofotômetro (modelo 6405, Jenway) a $535 \mathrm{~nm}$, usando como "branco" apenas o solvente. Para o cálculo do teor de antocianinas foi utilizada a seguinte fórmula (TEIXEIRA; STRINGHETA; OLIVEIRA, 2008):

Teor de antocianinas $\left(\mathrm{mg} 100 \mathrm{~g}^{-1}\right)=($ Abs $\mathrm{x} \mathrm{VE} 1 \mathrm{x}$ $\left.\mathrm{VE}_{2} \times 1.000\right) /($ Valq $\times \mathrm{m} \times 982$ ), sendo:

Abs: Absorbância;

$\mathrm{VE}_{1}$ : Volume total do extrato filtrado $(200 \mathrm{~mL})$;

$\mathrm{VE}_{2}$ : Volume total do extrato diluído $(100 \mathrm{~mL})$;

Valq: Aliquota da amostra filtrada a ser diluída (2 mL);

$\mathrm{m}$ : massa da amostra de uvas (g).
A quantificação do PT foi realizada baseado no método de Follin-Ciocauteau, de acordo com BucicKojic et al. (2007). Foi pesada, aproximadamente, $5 \mathrm{~g}$ de bagas de cada amostra, as quais foram trituradas com $50 \mathrm{~mL}$ de etanol a $50 \%$, durante 2 minutos, e realizada a centrifugação $(1.000 \mathrm{rpm})$ durante 5 minutos. Foram retiradas amostras de $0,2 \mathrm{~mL}$ desse extrato em tubo de ensaio, ao qual foram adicionados $1,8 \mathrm{~mL}$ de água destilada, $10 \mathrm{~mL}$ de solução de Follin-Ciocalteau a $10 \%$ e, entre 30 segundos a 8 minutos, adicionado $8 \mathrm{~mL}$ de solução de carbonato de sódio $\left(\mathrm{Na}_{2} \mathrm{CO}_{3}\right)$ a 7,5\%. O tubo foi agitado e mantido no escuro por 2 horas. A leitura foi feita em espectrofotômetro (modelo 6405, Jenway) a $765 \mathrm{~nm}$, usando como "branco" todos os reagentes sem a alíquota da amostra centrifugada, adicionando $2 \mathrm{~mL}$ de água destilada (completandose $0,2+1,8 \mathrm{~mL}$ ). O ácido gálico (GAE) foi usado como padrão e a curva de calibração confeccionada com solução de GAE, nas concentrações de 0,2; 0,$4 ; 0,6 ; 0,8 ; 1,0$ e $1,2 \mu \mathrm{g} \mathrm{mg}^{-1}$. A partir desta, foi obtido, por regressão linear, a equação: $y=a x+b$, na qual a variável "y" representava a absorbância, e desta forma, se obteve o valor do teor de composto fenólico em equivalente de ácido gálico.

Para o cálculo do PT foi empregada a seguinte fórmula (BUCIC-KOJIC et al., 2007):

PT $\left(\operatorname{mg~} 100 \mathrm{~g}^{-1}\right)=($ PTeq x V) x 10, sendo:

PTeq: valor obtido na equação da curva de calibração;

V: Diluição da amostra (50 mL/massa da amostra).

A cor das bagas foi analisada empregandose o colorímetro Minolta CR-10, sendo obtidas as variáveis de sua porção equatorial: $L^{*}$ (luminosidade) e $C^{*}$ (saturação), analisando-se 10 bagas por amostra (CANTIN et al., 2007).

Os dados obtidos foram submetidos à análise de variância e as médias comparadas pelo teste de Scott-Knott, a $5 \%$ de probabilidade. 


\section{Resultados e Discussão}

Não foram constatadas diferenças significativas entre os tratamentos em relação à massa e ao diâmetro das bagas, bem como da massa e o comprimento dos cachos (Tabela 1). Os resultados estão de acordo com os obtidos por Sato et al. (2009), e por Rizzon, Miele e Meneguzzo (2000), que caracterizam a uva 'Isabel' no Norte do Paraná e na Serra Gaúcha, respectivamente. Da mesma forma, Peppi, Fidelibus e Dokoozlian (2007a) não constataram diferenças na massa das bagas de 'Red Globe', com a aplicação de $S$-ABA. Porém, na uva 'Flame Seedless', a massa das bagas que receberam a aplicação de $S$-ABA foi superior (PEPPI; FIDELIBUS, 2008).

Tabela 1. Características físicas das bagas e dos cachos da videira 'Isabel' submetidas a diferentes tratamentos com S-ABA. Londrina, PR, 2013.

\begin{tabular}{lcccc}
\hline $\begin{array}{l}\text { Tratamentos } \\
\text { (Concentração em mg L }\end{array}$ & $\begin{array}{c}\text { Massa da } \\
\text { baga } \\
(\mathrm{g})\end{array}$ & $\begin{array}{c}\text { Diâmetro } \\
\text { da baga } \\
(\mathrm{mm})\end{array}$ & $\begin{array}{c}\text { Massa } \\
\text { do cacho } \\
(\mathrm{kg})\end{array}$ & $\begin{array}{c}\text { Comprimento } \\
\text { do cacho } \\
(\mathrm{cm})\end{array}$ \\
\hline Testemunha & 3,6 & 17,0 & 0,11 & 12,7 \\
$S$-ABA 200 (7DAV) & 3,6 & 16,7 & 0,12 & 12,9 \\
$S$-ABA 400 (7DAV) & 3,5 & 16,3 & 0,12 & 12,7 \\
$S$-ABA 200 (7DAV) + 200 (10DAC) & 3,3 & 16,3 & 0,11 & 12,1 \\
$S$-ABA 400 (7DAV) + 400 (10DAC) & 3,6 & 16,6 & 0,12 & 12,3 \\
\hline F & $0,8^{\text {ns }}$ & 1,2 ns & $1,0^{\text {ns }}$ & $1,8^{\text {ns }}$ \\
CV $(\%)$ & 8,0 & 3,4 & 8,1 & 11,8 \\
\hline
\end{tabular}

Médias seguidas da mesma letra nas colunas não diferem entre si pelo teste de $\operatorname{Scott} \operatorname{Knott}(p<0,05)$.

ns: não significativo. Obs: 7DAV = 7 Dias Após o Veraison; 10DAC $=10$ Dias Antes da Colheita.

Fonte: Elaboração dos autores.

Quanto às características químicas do mosto das bagas, foram verificadas diferenças em relação ao teor de sólidos solúveis (SS), acidez titulável (AT) e SS/AT (Tabela 2). Nos tratamentos com a aplicação de $S$-ABA foram observadas as maiores médias para o teor de SS e relação SS/AT, exceto para a concentração de $200 \mathrm{mg} \mathrm{L}^{-1}$, aplicada 7 dias após o veraison, em que não houve alteração química, comparativamente à testemunha. A aplicação de $S$-ABA proporcionou menor acidez, principalmente na concentração de $400 \mathrm{mg} \mathrm{L}^{-1}$, independente da época de aplicação. Cao et al. (2010), em experimento com uvas 'Kyoho', verificaram que a aplicação exógena de $S$-ABA proporcionou aumento no teor de SS. Entretanto, vários autores descreveram que a aplicação de $S$-ABA teve pouco ou nenhum efeito sobre os SS ou a AT das uvas (HAN; LEE; KIM, 1996; LEE et al., 1997; JEONG et al., 2004; PEPPI; FIDELIBUS; DOKGOZLIAN, 2006).
A concentração de antocianinas totais aumentou com aplicação de $S$-ABA, tanto nas bagas quanto nos sucos, sendo que, com $400 \mathrm{mg} \mathrm{L}^{-1}$ aplicado duas vezes (sete dias após o veraison e 10 dias antes da colheita) obteve-se a maior média para as bagas (Tabela 3). Independente das concentrações e das frequências de aplicação, no suco de uvas tratadas com $S$-ABA foram detectados os melhores resultados. Os resultados corroboram com os obtidos por Cao et al. (2010), com a aplicação de $250 \mathrm{mg} \mathrm{L}^{-1}$ de $S$-ABA na cv. Kyoho, assim como os verificados por Jeong et al. (2004), em que a aplicação de $S$-ABA estimulou o acúmulo de antocianinas na uva 'Cabernet Sauvingnon' duas a quatro semanas após a aplicação. A aplicação de $S$-ABA elevou o teor de antocianinas de outras cultivares, como 'Pionnier' (KONDO; MASUDA; INOUE, 1998) e 'Flame Seedless' (PEPPI; FIDELIBUS, 2008). Na 'Redglobe' foi 
observado que o $S$-ABA aumentou a quantidade total de antocianinas, sem afetar a composição dos pigmentos (PEPPI; FIDELIBUS; DOKOOZLIAN, 2007a). Ressalta-se que uma parte das antocianinas totais é perdida durante o processamento do suco e, consequentemente, interfere nos resultados da cor. Tal fato deve-se aos processos que a uva e o mosto são submetidos durante a produção do suco, tais como o aquecimento, a prensagem e a pasteurização (SISTRUNK; GASCOIGNE, 1983).

Tabela 2. Características químicas das bagas da videira 'Isabel' submetida a diferentes tratamentos com $S$-ABA. Londrina, PR, 2013.

\begin{tabular}{|c|c|c|c|}
\hline $\begin{array}{l}\text { Tratamentos } \\
\left.\text { (Concentração em } \mathrm{mg} \mathrm{L}^{-1}\right)\end{array}$ & $\begin{array}{c}\mathrm{SS}^{\mathrm{a} /} \\
\left({ }^{\circ} \mathrm{Brix}\right)\end{array}$ & $\begin{array}{c}\mathrm{AT}^{\mathrm{b} /} \\
(\% \text { de ácido tartárico })\end{array}$ & SS/AT \\
\hline Testemunha & $13,9 \mathrm{~b}$ & $1,14 \mathrm{a}$ & $12,2 \mathrm{~b}$ \\
\hline$S$-ABA 200 (7DAV) & $14,8 \mathrm{~b}$ & $0,93 \mathrm{~b}$ & $15,5 \mathrm{~b}$ \\
\hline$S$-ABA 400 (7DAV) & $15,7 \mathrm{a}$ & $0,83 \mathrm{c}$ & 18,9 a \\
\hline$S$-ABA $200(7 \mathrm{DAV})+200(10 \mathrm{DAC})$ & $16,9 \mathrm{a}$ & $0,95 \mathrm{~b}$ & $17,9 \mathrm{a}$ \\
\hline$S$-ABA $400(7 \mathrm{DAV})+400(10 \mathrm{DAC})$ & $15,4 \mathrm{a}$ & $0,78 \mathrm{c}$ & $20,1 \mathrm{a}$ \\
\hline $\mathrm{F}$ & $6,9^{*}$ & $8,9 *$ & $7,6^{*}$ \\
\hline CV (\%) & 5,9 & 9,9 & 13,4 \\
\hline
\end{tabular}

Médias seguidas da mesma letra nas colunas não diferem entre si pelo teste de $\operatorname{Scott} \operatorname{Knott}(p<0,05)$.

*: significativo $(\mathrm{p}<0,05) .{ }^{a}$ : sólidos solúveis. ${ }^{\mathrm{b} /}$ : acidez titulável. Obs: 7DAV $=7$ Dias Após o Veraison; $10 \mathrm{DAC}=10$ Dias Antes da Colheita.

Fonte: Elaboração dos autores.

Tabela 3. Antocianinas e polifenóis totais do mosto das bagas e do suco da videira 'Isabel' submetida a diferentes tratamentos com S-ABA. Londrina, PR, 2013.

\begin{tabular}{|c|c|c|c|c|}
\hline $\begin{array}{l}\text { Tratamentos } \\
\text { (Concentração em } \mathrm{mg} \mathrm{L}^{-1} \text { ) }\end{array}$ & $\begin{array}{l}\text { Antocianinas no } \\
\text { mosto das bagas } \\
\quad\left(\mathrm{mg} 100 \mathrm{~g}^{-1}\right)\end{array}$ & $\begin{array}{c}\text { Antocianina } \\
\text { suco } \\
\left(\mathrm{mg} \mathrm{L}^{-1}\right)\end{array}$ & $\begin{array}{c}\text { Polifenóis totais } \\
\text { no mosto das } \\
\text { bagas } \\
\left(\mathrm{mg} 100 \mathrm{~g}^{-1}\right)\end{array}$ & $\begin{array}{c}\text { Polifenóis totais } \\
\text { suco } \\
\left(\mathrm{g} \mathrm{L}^{-1}\right)\end{array}$ \\
\hline Testemunha & $3,6 \mathrm{~d}$ & $18,3 \mathrm{~b}$ & 41,2 & 0,2 \\
\hline$S$-ABA 200 (7DAV) & $6,4 \mathrm{c}$ & $42,3 \mathrm{a}$ & 39,3 & 0,3 \\
\hline$S$-ABA 400 (7DAV) & $9,5 \mathrm{~b}$ & $53,9 \mathrm{a}$ & 43,9 & 0,3 \\
\hline$S-\mathrm{ABA} 200(7 \mathrm{DAV})+200(10 \mathrm{DAC})$ & $8,4 \mathrm{~b}$ & $38,2 \mathrm{a}$ & 38,7 & 0,3 \\
\hline$S-\mathrm{ABA} 400(7 \mathrm{DAV})+400(10 \mathrm{DAC})$ & $12,2 \mathrm{a}$ & $57,5 \mathrm{a}$ & 42,5 & 0,3 \\
\hline $\mathrm{F}$ & $14,3 *$ & $6,8^{*}$ & $0,6^{\mathrm{ns}}$ & $1,9^{\mathrm{ns}}$ \\
\hline CV $(\%)$ & 21,2 & 28,2 & 13,2 & 13,2 \\
\hline
\end{tabular}

Médias seguidas da mesma letra nas colunas não diferem entre si pelo teste de $\operatorname{Scott} \operatorname{Knott}(p<0,05)$. *: significativo ( $<<0,05)$. Obs: 7DAV $=7$ Dias Após o Veraison; 10DAC $=10$ Dias Antes da Colheita.

Fonte: Elaboração dos autores.

Para o índice de polifenóis totais (PT) não houve diferença estatística entre os tratamentos (Tabela 3). A maioria dos trabalhos realizados está baseada na quantificação destes compostos nos vinhos, sendo raras as informações sobre o PT nas bagas das uvas e no suco (SATO et al., 2012). Observa-se, porém, que as médias neste trabalho estão abaixo das obtidas por Soares et al. (2008), que registraram PT de $163,73 \pm 12,4 \mathrm{mg} 100 \mathrm{~g}^{-1}$, para a 'Isabel' cultivada no Vale do Paraíba-SP.

Em relação aos atributos de cor das bagas (Tabela 4), verificou-se que os tratamentos em que o $S$-ABA foi aplicado duas vezes, nas duas concentrações (200 e $400 \mathrm{mg} \mathrm{L}^{-1}$ ), bem como aplicado uma vez 
na concentração de $400 \mathrm{mg} \mathrm{L}^{-1}$, resultaram nas menores médias de $L^{*}$ (entre 24,6 à 25,2), o que indica que as bagas de 'Isabel' submetidas a esses tratamentos apresentaram cor mais escura. Observações semelhantes foram feitas por Peppi, Fidelibus e Dokoozlian (2007a), em 'Red Globe'; Peppi e Fidelibus (2008), em 'Flame Seedless'; e Peppi, Walker e Fidelibus (2008), em 'Crimson
Seedless', na Califórnia, EUA. Peppi, Fidelibus e Dokoozlian (2007b) descreveram que, devido à alta relação linear entre o $L^{*}$ e o teor de antocianinas nas bagas da videira 'Crimson Seedless', avaliações de cor utilizando essa variável deve ser preferida para se determinar o efeito de práticas culturais na cor das uvas.

Tabela 4. Atributos de cor das bagas da videira 'Isabel' submetida a diferentes tratamentos com $S$-ABA. Londrina, PR, 2013.

\begin{tabular}{lcc}
\hline $\begin{array}{l}\text { Tratamentos } \\
\text { (Concentração em mg L-1) }\end{array}$ & $L^{*}$ \\
\hline Testemunha & $27,9 \mathrm{a}$ & $4,7 \mathrm{a}$ \\
S-ABA 200 (7DAV) & $26,4 \mathrm{~b}$ & $3,3 \mathrm{~b}$ \\
S-ABA 400 (7DAV) & $25,2 \mathrm{c}$ & $1,8 \mathrm{c}$ \\
S-ABA 200 (7DAV) + 200 (10DAC) & $25,5 \mathrm{c}$ & $2,4 \mathrm{c}$ \\
$S$-ABA 400 (7DAV) + 400 (10DAC) & $24,6 \mathrm{c}$ & $1,8 \mathrm{c}$ \\
\hline F & $12,3^{*}$ & $8,5^{*}$ \\
CV $(\%)$ & 2,9 & 30,0 \\
\hline
\end{tabular}

Médias seguidas da mesma letra nas colunas não diferem entre si pelo teste de Scott Knott $(p<0,05)$. ns: não significativo. *: significativo $(p<0,05) . L^{*}$ : luminosidade; $C^{*}$ : chroma. Obs: 7DAV $=7$ Dias Após o Veraison; 10DAC $=10$ Dias Antes da Colheita.

Fonte: Elaboração dos autores.

Para as uvas destinadas à elaboração de suco, dentre os vários aspectos que devem ser considerados, destacam-se o teor de açúcar, que deve ser alto, porém equilibrado com a acidez e o teor de matéria corante, que também deve ser elevado, por estar diretamente relacionado à aceitabilidade do suco por parte do consumidor (ASSIS et al., 2011). Em avaliação sensorial de suco de uva 'Isabel', em cortes com diferentes cultivares, Borges et al. (2011) verificaram que, para o atributo cor, o suco que continha somente uva 'Isabel' obteve a menor aceitação pelo grupo de provadores, devido à cor menos acentuada.

Em relação à variável $C^{*}$ (índice Chroma), que define a saturação de cor, foram observadas diferenças significativas (Tabela 4). Os tratamentos com aplicação de $S$-ABA apresentaram as menores médias de $C^{*}$, ou seja, o menor valor de saturação. De acordo com Piva, Lopez e Morgan, (2006), a
'Isabel' é considerada uma uva preta e, neste caso, os valores cromáticos inferiores dos cachos tratados com $S$-ABA representam cor mais escura das bagas. Em experimento conduzido por Peppi, Fidelibus e Dokgozlian (2006), com a videira 'Flame Seedless', a aplicação de $S$-ABA e ethephon, no veraison, acarretou em menores médias de $C^{*}$, assim como na uva 'Crimson Seedless', em trabalho de Peppi, Walker e Fidelibus (2008).

Com base no exposto, pôde-se verificar que a utilização de $S$-ABA exerce efeito sobre a melhoria na concentração de antocianinas das bagas e do suco da uva 'Isabel', principalmente com $400 \mathrm{mg} \mathrm{L}^{-1}$ aos sete dias após o veraison e aos 10 dias antes da colheita. Além disso, os tratamentos com a aplicação de $S$-ABA (400 $\mathrm{mg} \mathrm{L}^{-1}$ nas duas épocas e $200 \mathrm{mg} \mathrm{L}^{-1}$ aplicados sete dias após o veraison) propiciaram o aumento no teor de SS e relação SS/AT e a redução na acidez das bagas. Desse modo, o uso do $S$-ABA 
é uma alternativa promissora, que implica no incremento do valor comercial do produto, visto que possibilita que o suco de uva 'Isabel' possua a cor mais intensa, sem a necessidade de ser cortado com o suco de cultivares tintureiras.

\section{Conclusões}

1) A aplicação de $S$-ABA não altera as características físicas da uva 'Isabel'.

2) O regulador $S$-ABA aumenta a relação $\mathrm{SS} / \mathrm{AT}$ das bagas, exceto na concentração de $200 \mathrm{mg} \mathrm{L}^{-1}$ aplicada 7 dias após o veraison.

3) Maior concentração de antocianinas nas bagas e melhoria nos atributos de cor são obtidos com $S$-ABA na concentração de $400 \mathrm{mg} \mathrm{L}^{-1}$ aplicado duas vezes, aos 7 dias após o veraison e aos 10 dias antes da colheita.

4) Aplicações de $S$-ABA resultam em maior teor de antocianina no suco.

\section{Referências}

ABE, L. T.; MOTA, R. V.; LATOJO, F. M.; GENOVESE, M. I. Compostos fenólicos e capacidade antioxidante de cultivares de uvas Vitis labrusca L. e Vitis vinifera L. Ciência Tecnologia Alimentos, Campinas, v. 27, n. 2, p. 394-400, 2007.

ASSIS, A. M. D.; YAMAMOTO, L. Y.; SOUZA, F. S. D.; BORGES, R. D. S.; ROBERTO, S. R. Evolução da maturação e características físico-químicas e produtivas das videiras 'BRS Carmem' e 'Isabel'. Revista Brasileira Fruticultura, Jaboticabal, v. 33, n. 1, p. 493-498, 2011.

BORGES, R. D. S.; PRUDENCIO, S. H.; ROBERTO, S. R.; ASSIS, A. M. D. Avaliação sensorial de suco de uva cv. Isabel em cortes com diferentes cultivares. Revista Brasileira Fruticultura, Jaboticabal, v. 33, n. 1, p. 584591, 2011.

BUCIC-KOJIC, A.; PLANINIC, M.; TOMAS, S.; BILIC, M.; VELIC, D. Study of solid-liquid extraction kinetics of total polyphenols from grapes seeds. Journal of Food Engineering, Davis, v. 81, n. 1, p. 236-242, 2007.

CANTÍN, C. M. A.; FIDELIBUS, B. M. W.; CRISOSTO, C. H. Application of abscisic acid (ABA) at veraison advanced red color development and maintained postharvest quality of 'Crimson Seedless' grapes. Postharvest Biology and Technology, Amsterdam, v. 46, n. 3, p. 237-241, 2007.

CAO, M.; BAI, X.; LI, Y.; XIE, T.; WEN, R.; LIU, J. Effect of abscisic acid on the color and fruit quality of Kyoho grape. Guangdong Agricultural Sciences, Guiyang, v. 2, n. 39, p. 111-113, 2010.

CAVIGLIONE, J. H.; KIIHL, L. R. B.; CARAMORI, P. H.; OLIVEIRA, D. Cartas climáticas do Paraná. Londrina: IAPAR, 2000. CD-ROM. Disponível em: $<$ http://www.iapar.br/modules/conteudo/conteudo. php? conteudo=677> . Acesso em: 04 jun. 2012.

HAN, D. H.; LEE, S. M.; KIM, S. B. Effects of ABA and ethephon treatments on coloration and fruit quality in Kyoho grape. Journal of the American Society for Horticultural Science, Alexandria, v. 37, n. 3, p. 416-420, 1996.

INSTITUTO ADOLFO LUTZ - IAL. Normas analíticas do Instituto Adolfo Lutz: métodos químicos e físicos para análise dos alimentos. 3. ed. São Paulo: Instituto Adolfo Lutz, 1985. 371 p.

JEONG, S. T.; UOTO, N. G.; KOBAYASHI, S.; ESAKA, M. Effects of plant hormones and shading on the accumulation of an-and the expression of anthocyanin biosynthetic genes iberry skins. Plant Science, London, v. 167, n. 2, p. 247-252, 2004.

JUBILEU, B. S.; SATO, A. J.; ROBERTO, S. R. Caracterização fenológica e produtiva das videiras 'Cabernet Sauvignon' e 'Alicante' (Vitis vinifera L.) produzidas fora de época no Norte do Paraná. Revista Brasileira de Fruticultura, Jaboticabal, v. 32, n. 2, p. 451-462, 2010.

KISHINO, A. Y.; CARVALHO, S. L. C.; ROBERTO, S. R. Viticultura tropical: o sistema de produção do Paraná. Londrina: IAPAR, 2007. 366 p.

KONDO, S.; MASUDA, E.; INOUE, K. Relation between ABA application and fruit quality of 'Pionnier' grape (Vitis sp.). Acta Horticulturae, Wageningen, v. 464, n. 130, p. 35-40, 1998.

KOYAMA, R.; ASSIS, A. M. D.; YAMAMOTO, L. Y.; BORGES, W. F. S.; PRUDENCIO, S. H.; ROBERTO, S. R. Exogenous abscisic acid increases the anthocyanin concentration of berry and juice from 'Isabel' grapes (Vitis labrusca L.). HortScience, Alexandria, v. 49, p. 460-464, 2014.

LEE, D. H.; FRANCIS, F. J. Standardization of pigment analyses in cranberries. HortScience, Stanford, v. 7, n. 1, p. 83-84, 1972. 
LEE, K. S.; LEE, J. C.; HWANG,Y. S.; HUR, I. B. Effects of natural type (S)-(+)- abscisic acid on anthocyanin accumulation and maturity in 'Kyoho' grapes. Korean Journal of Horticultural Science \& Technology, Suwon, v. 38, n. 6, p. 717-721, 1997.

MAIA, J. D. G.; CAMARGO, U. A. Produção de uva Isabel para processamento, no sistema GDC, em região tropical do Brasil. Bento Gonçalves: Embrapa Uva e Vinho, 2007. 13 p. (Comunicado técnico, 79). Disponível em: <http://www.cnpuv.embrapa.br/publica/ comunicado/cot079.pdf $>$. Acesso em: 14 maio 2012.

MELLO, L. M. R. Vitivinicultura brasileira: Panorama 2011. Bento Gonçalves: EMBRAPA, 2012. 4 p. (Comunicado técnico, 125).

PEPPI, M. C.; FIDELIBUS, M. W.; DOKGOZLIAN, N. Abscisic acid application timing and concentration affect firmness, pigmentation and color of 'Flame Seedless' grapes. HortScience, Califórnia, v. 41, n. 4, p. 14491445, 2006.

. Application timing and concentration of abscisic acid affect the quality of 'Redglobe' grapes. Journal of Horticultural Science and Biotechnology, United Kingdom, v. 82, n. 2, p. 304-310, 2007a.

Timing and concentration of abscisic acid applications affect the quality of 'Crimson Seedless' grapes. International Journal of Fruit Science, Binghamton, v. 7, n. 4, p. 71-83, 2007b.

PEPPI, M. C.; FIDELIBUS M. W. Effects of forchlorfenuron and abscisic acid on the quality of 'Flame Seedless' grapes. Hortscience, Califórnia, v. 43, n. 1, p. 173-176, 2008.

PEPPI, M. C.; WALKER, M. A.; FIDELIBUS, M. W. Application of abscisic acid rapidly upregulated UFGT gene expression and improved color of grape berries. Vitis, Frankfurt, v. 47, n. 1, p. 11-14, 2008.

PIVA, C. R.; LOPEZ G. J. L.; MORGAN, W. The ideal table grapes for the Spanish market. Revista Brasileira de Fruticultura, Jaboticabal, v. 28, n. 2, p. 258-261, 2006.

PROTAS, J. F. S.; CAMARGO, A. Vitivinicultura brasileira: panorama setorial de 2010. IBRAVIN: Embrapa Uva e Vinho, Bento Gonçalves, 2011. 110 p.

RIBICHAUD, J. L.; NOBLE, A. C. Astringency and bitterness of selected phenolic in wines. Journal of the Science of Food and Agriculture, Inglaterra, v. 53, n. 3, p. 343-353, 1990.

RIZZON, L. A.; MIELE, A.; MENEGUZZO, J. Avaliação da uva cv. Isabel para elaboração de vinho tinto. Ciência Tecnologia de Alimentos, Campinas, v. 20, n. 1, p. 115$121,2000$.
ROBERTO, S. R.; ASSIS, A. M.; YAMAMOTO, L. Y.; MIOTTO, L. C. V.; SATO, A. J.; KOYAMA, R.; GENTA, $\mathrm{W}$. Application timing and concentration of abscisic acid improve color of 'Benitaka' table grape. Scientia Horticulturae, Amsterdam, v. 142, p. 44-48, 2012.

ROBERTO, S. R.; ASSIS, A. M.; YAMAMOTO, L. Y.; MIOTTO, L. C. V.; KOYAMA, R.; SATO, A. J.; BORGES, R. D. S. Ethephon use and application timing of abscisic acid for improving color of 'Rubi' table grape. Pesquisa Agropecuária. Brasileira, Brasília, v. 48, n. 7, p. 797-800, 2013 .

SATO, A. J.; JUBILEU, B. S.; SANTOS, C. E.; BERTOLUCCI, R.; SILVA, R. A. L.; CARIELO, M.; GUIRAUD, M. C.; FONSECA, I. C. B.; ROBERTO, S. R. Fenologia e demanda térmica das videiras Isabel e Rubea sobre diferentes porta-enxertos na região norte do Paraná. Semina: Ciências Agrárias, Londrina, v. 29, n. 2, p. 283-292, 2008.

SATO, A. J.; JUBILEU, B. S.; SANTOS, C. E.; BERTOLUCCI, R.; SILVA, R. A. L.; CARIELO, M. ; GUIRAUD, M. C.; FONSECA, I. C. B.; ROBERTO, S. R. Evolução da maturação e características fisico-químicas de uvas da cultivar Isabel sobre diferentes porta-enxertos na região Norte do Paraná. Semina, Ciências Agrárias, Londrina, v. 30, n. 1, p. 11-20, 2009.

SATO, A. J.; ROSA, C. I. L. F.; MENEZES, D.; KWIATKOWSK, A.; CLEMENTE, E.; ROBERTO, S. R. Caracterização fenólica das uvas 'Alicante' e 'Syrah' cultivadas em safra fora de época. Revista Brasileira de Fruticultura, Jaboticabal, v. 34, n. 1, p. 116-123, 2012.

SISTRUNK, W. A.; GASCOIGNE, H. L. Stability of color in concord grape juice and expression of color. Journal of Food Science, Chicago, v. 48, n. 2, p. 430435, 1983.

SOARES, M.; WELTER, L.; KUSKOSKI, E. M.; GONZAGA, L.; FETT, R. Compostos fenólicos e atividade antioxidante da casca de uvas Niágara e Isabel. Revista Brasileira de Fruticultura, Jaboticabal, v. 30, n. 1, p. 59-64, 2008.

TEIXEIRA, L. N.; STRINGHETA, P. C.; OLIVEIRA, F. A. Comparação de métodos para quantificação de antocianinas. Revista Ceres, Viçosa, v. 55, n. 4, p. 297 304, 2008. 
